\title{
Modelling stochastic correlation
}

\author{
Long Teng ${ }^{*}$, Matthias Ehrhardt and Michael Günther
}

\author{
${ }^{*}$ Correspondence: \\ teng@math.uni-wuppertal.de \\ Lehrstuhl für Angewandte \\ Mathematik und Numerische \\ Analysis, Fakultät für Mathematik \\ und Naturwissenschaften, \\ Bergische Universität Wuppertal, \\ Gaußstraße 20, Wuppertal, 42119 , \\ Germany
}

\begin{abstract}
This work deals with the stochastic modelling of correlation in finance. It is well known that the correlation between financial products, financial institutions, e.g., plays an essential role in pricing and evaluation of financial derivatives. Using simply a constant or deterministic correlation may lead to correlation risk, since market observations give evidence that the correlation is hardly a deterministic quantity. For example, we illustrate this issue with the analysis of correlation between daily returns time series of S\&P Index and Euro/USD exchange rates.

The approach of modelling the correlation as a hyperbolic function of a stochastic process has been recently proposed. Here, we review this novel concept and generalize this approach to derive stochastic correlation processes (SCP) from a hyperbolic transformation of the modified Ornstein-Uhlenbeck process. We determine a transition density function of this SCP in closed form which could be used easily to calibrate SCP models to historical data.

As an illustrating example of our new approach, we compute the price of a quantity adjusting option (Quanto) and discuss concisely the effect of considering stochastic correlation on pricing the Quanto.
\end{abstract}

Keywords: stochastic correlation; quanto option; correlation risk; Ornstein-Uhlenbeck process; transition density; hyperbolic tangent function

\section{Introduction}

Correlation is a well established concept for quantifying the relationship between financial assets. It plays an essential role in several financial applications, e.g. the arbitrage pricing model [1] is based on correlation as a measure for the dependence of assets. Also in portfolio credit models, the default correlation is one fundamental factor of risk evaluation, see e.g. [2] and [3].

For two random variables $X_{1}$ and $X_{2}$ with finite variances, the correlation of them is defined as

$$
\rho_{1,2}=\operatorname{Corr}\left(X_{1}, X_{2}\right)=\frac{\operatorname{Cov}\left(X_{1}, X_{2}\right)}{\sigma_{1} \sigma_{2}},
$$

with the covariance

$$
\operatorname{Cov}\left(X_{1}, X_{2}\right)=\mathbb{E}\left[\left(X_{1}-\mu_{1}\right)\left(X_{2}-\mu_{2}\right)\right]
$$

where $\mu_{i}$ and $\sigma_{i}$ are the expectation and standard deviation of $X_{i}, i=1,2$. Here $\rho_{1,2}$ denotes a coefficient number in the interval $[-1,1]$. The boundaries -1 and 1 will be reached if and

(c) 2016 Teng et al. This article is distributed under the terms of the Creative Commons Attribution 4.0 International License (http://creativecommons.org/licenses/by/4.0/), which permits unrestricted use, distribution, and reproduction in any medium, provided you give appropriate credit to the original author(s) and the source, provide a link to the Creative Commons license, and indicate if changes were made. 
only if $X_{1}$ and $X_{2}$ are indeed linearly related. The greater the absolute value of $\rho_{1,2}$ the stronger the dependence between $X_{1}$ and $X_{2}$ is.

Generally, there are several disadvantages or fallacies of the correlation concept (1), we state only some of them:

- If the random variables $X_{1}$ and $X_{2}$ are independent, then it follows $\rho_{1,2}=0$. However, the converse implication does not hold, since in (1) only the two first moments are included. For example, we compute $\rho_{1,2}=0$ for $X_{2}=X_{1}^{2}$. Indeed, $X_{1}$ and $X_{2}$ depend even almost perfectly on each other. This illustrates that the correlation coefficient only recognizes linear dependencies between random variables.

- Correlation is invariant under strictly increasing linear transformations, but, in contrast to Copula methods, not invariant under nonlinear strictly increasing transformations. For example, in general the correlation of the random variables $X_{1}$ and $X_{2}$ does not equal the correlation of the random variables $\ln X_{1}$ and $\ln X_{2}$, i.e. after a transformation of the financial data the correlation may change.

- Usually, the given marginal distributions and pairwise correlations of a random vector cannot determine its joint distribution.

- Finally, as stated above, the variances of the two random variables $X_{1}$ and $X_{2}$ has to be finite. This assumptions is not fulfilled for every standard distribution, e.g. the Student's $t$-distribution with $v \leq 2$ possess an infinite variance.

For more detailed information about the disadvantages or fallacies we refer to [4]. Although this concept of correlation (1) to measure dependence inherits several limitations, it has been widely applied in financial applications.

In financial markets, the first problem of using a correlation concept is the observability. Unlike prices, volatilities, exchange rates and so on, the correlation cannot be observed directly in the market and can only be measured in the context of a model. The easiest estimator of the correlation is the sample correlation coefficient. Given a series of $N$ measurements of $X_{1}$ and $X_{2}$, which are observable quantities in the market, and denoting the measurements by $x_{1, j}$ and $x_{2, j}, j=1,2, \ldots, N$, the sample coefficient correlation reads

$$
\hat{\rho}_{12}=\frac{\sum_{j=1}^{N}\left(x_{1, j}-\bar{\mu}_{1}\right)\left(x_{2, j}-\bar{\mu}_{2}\right)}{\sqrt{\sum_{j=1}^{N}\left(x_{1, j}-\bar{\mu}_{1}\right)^{2} \sum_{j=1}^{N}\left(x_{2, j}-\bar{\mu}_{2}\right)^{2}}},
$$

where $\bar{\mu}_{1}$ and $\bar{\mu}_{2}$ are the sample means of $X_{1}$ and $X_{2}$.

In financial models, stochastic processes are used quite often to model data series, like price, interest rate and exchange rate. When considering diffusion processes, the dependence between the series is given by correlated Brownian motions. Two Brownian motions $W_{1}$ and $W_{2}$ are correlated by the symbolic notion

$$
d W_{1, t} d W_{2, t}=\rho_{1,2} d t
$$

For example, in the multivariate Black-Scholes model, the correlation of the log-returns is used as a measure of the dependence between asset processes. A further example of coupled stochastic processes appears when pricing a quantity adjusting option (Quanto) in the framework of the Black-Scholes model:

$$
\left\{\begin{array}{l}
d S_{t}=\mu_{S} S_{t} d t+\sigma_{S} S_{t} d W_{t}^{S}, \\
d R_{t}=\mu_{R} R_{t} d t+\sigma_{R} R_{t} d W_{t}^{R},
\end{array}\right.
$$


with positive constants $\mu_{S}, \mu_{R}, \sigma_{S}$ and $\sigma_{R}$. The first stochastic differential equation (SDE) describes the price, $S_{t}$ of the traded asset in a currency A. The second SDE is used to model the exchange rate $R_{t}$ between currency $\mathrm{A}$ and another currency $\mathrm{B}$. Besides, the Brownian motions are assumed to be correlated by a constant correlation $\rho \in[-1,1]$ which is a measure of co-movements between $S_{t}$ and $R_{t}$.

As we explained above, the constant correlation coefficient defined by (1) only captures linear relationships between $X_{1}$ and $X_{2}$. Therefore, in the model (5) a linear dependence between $S_{t}$ and $R_{t}$ is assumed. However, from the market we realize that there is often a nonlinear dependence between $S_{t}$ and $R_{t}$. Specifically, a constant correlation means that the two return processes are jointly stationary which is generally not true in the real world. Thus, the dependence can be hardly modelled by a fixed constant, i.e. the constant correlation may not be an appropriate measure of co-dependence. Using constant ("wrong") correlation may result some 'correlation risk'. There exist already some works which show that the correlation should not be constant and even changes over a small time interval as the volatility, see e.g. [5]. Several approaches generalize the constant correlation to a time-varying and stochastic concept, like Dynamic Conditional Correlation model in [6], Local correlation models see e.g. [7] and the Wishart autoregressive process proposed by Gourieroux [8] that guarantees the positive definiteness of the variance-covariance matrix.

In fact, either implied correlation in the context of a model or historical correlation from the market data show us that the correlation should be time-varying and behave like a stochastic process. To illustrate this statement, we make an example of historical correlations between SEP 500 index and Euro/US-Dollar exchange rate on a daily basis. We use $\bar{s}$ and $\bar{r}$ to denote the daily return series of S\&P 500 and Euro/US-Dollar exchange rate and fix a time window $n_{T}$, e.g. $n_{T}=60$ for 60 -day historical correlation. At time $t$, using the $n_{T}$ times most recent daily returns, the correlation at time $t$ is given by the following estimator

$$
\hat{\rho}_{t}=\frac{\sum_{j=1}^{n_{T}}\left(\hat{s}_{t-j}-\frac{1}{n_{T}} \sum_{j=1}^{n_{T}} \hat{s}_{t-j}\right)\left(\hat{r}_{t-j}-\frac{1}{n_{T}} \sum_{j=1}^{n_{T}} \hat{r}_{t-j}\right)}{\sqrt{\sum_{j=1}^{n_{T}}\left(\hat{s}_{t-j}-\frac{1}{n_{T}} \sum_{j=1}^{n_{T}} \hat{s}_{t-j}\right)^{2} \sum_{j=1}^{n_{T}}\left(\hat{r}_{t-j}-\frac{1}{n_{T}} \sum_{j=1}^{n_{T}} \hat{r}_{t-j}\right)^{2}}} .
$$

Then we roll it to the time $t+1$, and so on to obtain a series of correlations through the time. The 15-day, 30-day and 60-day historical correlations are displayed in Figure 1.

We observe that the longer a time window (the value of $n_{T}$ ) the less volatile a historical correlation is. In Figure 1, the 15-day historical correlation is more variable than the 30day historical correlation which is again more variable than the 60-day correlation. With a longer averaging period a long-term correlation is calculated. If we choose $n_{T}=10$ or 15 days, the estimated correlation for each time $t$ using (6), could be seen as a short-term correlation of the current market phenomena whose immediate past returns are used for the estimation. It is worthwhile noting that the events, especially, some extreme events in a time window will affect the correlation which would be estimated in the following time windows, even has a delayed effect on the long-term correlation.

If one assumes that the phenomena in the past could have a reflection in the future, one would like to use the historical correlation as a forecast for the future. It could be a better way for correlation forecasting, if one describes the correlation using a meanreverting stochastic process. Besides, modelling correlation as a stochastic process, not only 


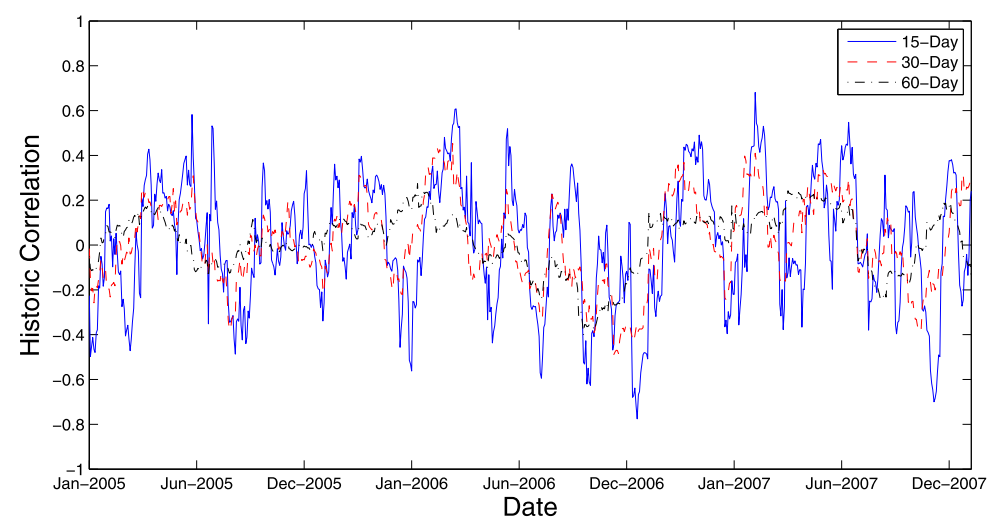

Figure 1 Historical correlation between S\&P 500 and Euro/US-Dollar exchange rate, source of data: www.yahoo.co.

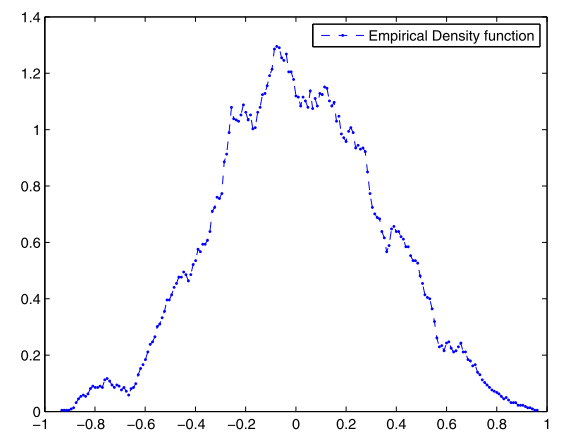

(a) bandwidth $1 / 40$

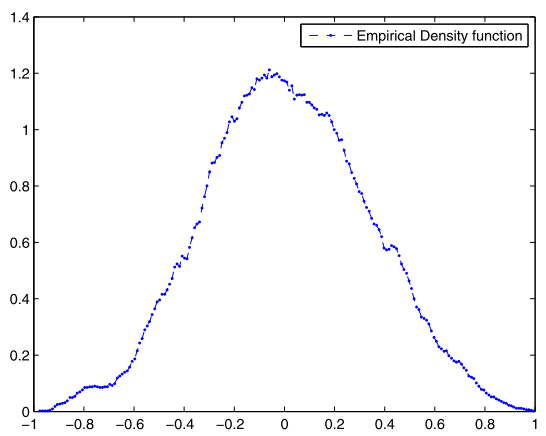

(b) bandwidth $1 / 30$

Figure 2 Empirical density function of the historical correlation between S\&P 500 and Euro/US-Dollar exchange rate.

the variation of the short-term correlation can be reflected, also the attributes of long-term correlation is determined by the long-term parameter values, like long-term mean value and mean reversion speed.

To see more properties, which a mean-reverting stochastic process should have to be a SCP, we plot its empirical density functions in Figure 2 using different bandwidths. We refer to [9] for details about the estimation of a density function from historical data.

From studying the empirical density functions we require that the stochastic correlation process should satisfy the following properties:

(i) only takes values in the interval $(-1,1)$,

(ii) varies around a mean value,

(iii) the probability mass tends to zero at the boundaries $-1,+1$.

One stochastic correlation process was proposed by van Emmerich [10], including a restriction on the parameter range to ensure that the boundaries -1 and 1 of the correlation process are not attractive and unattainable. A modified Jacobi process is suggested in [11] modelling stochastic correlation. A more general stochastic correlation process was proposed by Teng et al. [12], which relies on the hyperbolic transformation with the hyper- 
bolic tangent function of any mean-reverting process with positive and negative values, the properties (i)-(iii) above can be thus directly satisfied without facing any additional parameter restrictions. Hence, the subsequent calibration process is much simpler.

In this work, we study the general SCP by Teng et al. [12]. We show that the correlation process by van Emmerich can be obtained by this general method, i.e. the correlation process by van Emmerich turns out to be a special case of the hyperbolic transformation of a stochastic process. Furthermore, we apply this general approach to find a new SCP which has a transition density function in closed form. Finally, as an illustrating example, we compute the price of a Quanto under stochastic correlation by our new SCP and investigate the effect of considering stochastic correlation on pricing the Quanto.

\section{A general stochastic correlation model}

Here we study the hyperbolic transformation proposed in [12] of a mean-reverting process to be a correlation process. We show that the correlation process model of van Emmerich [10] can be obtained by transforming a mean-reverting process with the hyperbolic tangent function. We fix a probability space $(\Omega, \mathcal{F}, \mathbb{P})$ and an information filtration $\left(\mathcal{F}_{t}\right)_{t \in \mathbb{R}^{+}}$ satisfying the usual conditions, see e.g. [13].

\subsection{The transformed mean-reverting process}

For the motivations and the properties (i)-(iii) in Section 1, Teng et al. [12] proposed the hyperbolic tangent function of a mean-reverting stochastic process $X_{t}$, like the OrnsteinUhlenbeck process [14] or the square root diffusion processes (with positive and negative values)

$$
d X_{t}=a\left(t, X_{t}\right) d t+b\left(t, X_{t}\right) d W_{t}, \quad t \geq 0, X_{0}=x_{0}
$$

to model the correlations as

$$
\rho_{t}=\tanh \left(X_{t}\right), \quad \rho_{0}=\tanh \left(x_{0}\right) \in(-1,1) .
$$

Obviously, the properties (i)-(iii) are fulfilled due to the range of values of the hyperbolic tangent function and mean reversion of the process. Besides, the function tanh is symmetrical and measurable. Although the function tanh can not really attain -1 and 1 which presents perfect negative and perfect positive dependence, respectively. It should make no difference to use this function for modelling correlations, because the correlation equal to -1 or 1 is indeed an extreme event which happens very rarely in the real market, see e.g. Figure 1. Besides, the function tanh tends to the boundaries -1 and 1 at infinity.

Applying Itồs Lemma with (8)

$$
d \rho_{t}=d \tanh \left(X_{t}\right)=\frac{\partial \tanh \left(X_{t}\right)}{\partial t} d t+\frac{\partial \tanh \left(X_{t}\right)}{\partial x} d X_{t}+\frac{1}{2} \frac{\partial^{2} \tanh \left(X_{t}\right)}{\partial x^{2}}\left(d X_{t}\right)^{2},
$$

we obtain the stochastic correlation process (SCP)

$$
d \rho_{t}=\left(1-\rho_{t}^{2}\right)\left(\left(\tilde{a}-\rho_{t} \tilde{b}^{2}\right) d t+\tilde{b} d W_{t}\right), \quad t \geq 0,
$$

where $\rho_{0} \in(-1,1), \tilde{a}=a\left(t, \operatorname{artanh}\left(\rho_{t}\right)\right)$ and $\tilde{b}=b\left(t, \operatorname{artanh}\left(\rho_{t}\right)\right)$. From (10) we see that there is a suitable number of free parameters to calibrate the model to market data. Besides, it 
is obvious that, in this approach any mean-reverting process (with positive and negative values) can be considered without facing any additional parameter restrictions. The free parameters are hidden in the functions $a$ and $b$, see the example (13) in Section 2.3 and (20) in Section 3.1.

\subsection{Transformation with other functions}

Although we could intuitively observe that the function $\tanh (x)$ is eminently suitable for correlation modelling, one can still ask whether other functions having values inside the interval $(-1,1)$, like trigonometric functions or $\frac{2}{\pi} \arctan \left(\frac{\pi}{2} x\right), x \in \mathbb{R}$ can also be applied for this purpose? In theory, such functions could be used for the SCP model above. However, the trigonometric function is a periodic function, the arising complex number will complicate further calculations. For the function $\frac{2}{\pi} \arctan \left(\frac{\pi}{2} x\right)$, its Itô's formula for (7) is given by

$$
\begin{aligned}
d \rho_{t}=d \frac{2}{\pi} \arctan \left(\frac{\pi}{2} X_{t}\right)= & \left(\frac{\tilde{a}}{\left(1+\tan ^{2}\left(\frac{\rho_{t} \pi}{2}\right)\right)}-\frac{\pi \tilde{b}^{2} \tan \left(\frac{\rho_{t} \pi}{2}\right)}{2\left(1+\tan ^{2}\left(\frac{\rho_{t} \pi}{2}\right)\right)^{2}}\right) d t \\
& +\frac{\tilde{b}}{\left(1+\tan ^{2}\left(\frac{\rho_{t} \pi}{2}\right)\right)} d W_{t},
\end{aligned}
$$

which is rather complicate such that the further computation will be tedious. Furthermore, we compare the function $\frac{2}{\pi} \arctan \left(\frac{\pi}{2} x\right)$ with $\tanh (x)$ in Figure 3 and find that the both function are close to each other in the neighbourhood of $x=0$. However, compared with $\tanh (x)$, the function $\frac{2}{\pi} \arctan \left(\frac{\pi}{2} x\right)$ grows much slower up to 1 and down to -1 , so that the estimation of the correlation will be worsened. The reason is similar to the estimation for the heavy tailed distributions.

\subsection{The correlation model of van Emmerich}

As an example, we show that the correlation model of van Emmerich can be obtained by transforming the special mean-reverting process (12), i.e. the van Emmerich's correlation

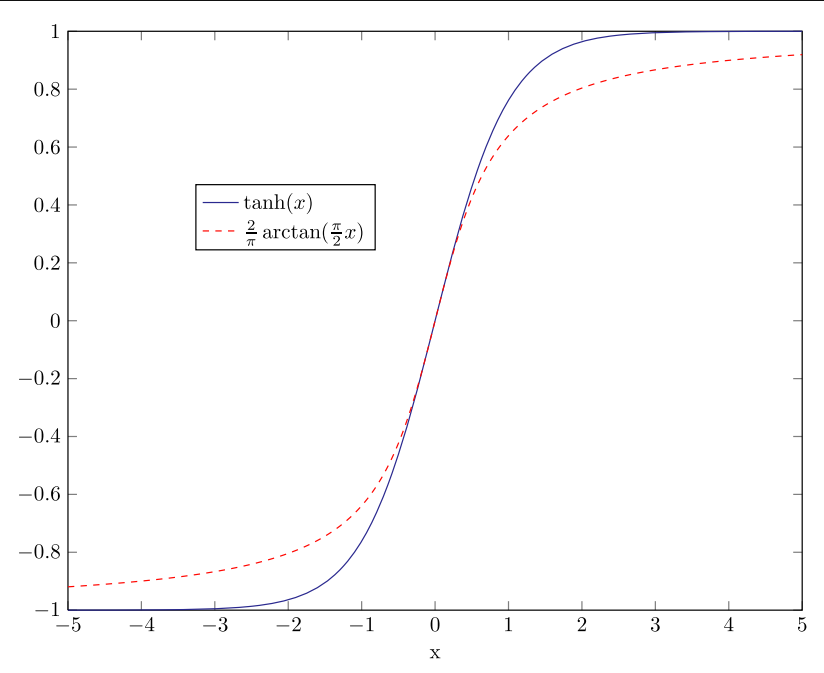

Figure 3 Comparison of $\tanh (x)$ and $\frac{2}{\pi} \arctan \left(\frac{\pi}{2} x\right)$ : the later is less steep having larger tails. 
process is just a special case of the general transformation [12]. To do so, we define the following mean-reverting process

$$
d X_{t}=\frac{\kappa\left(\mu-\tanh \left(X_{t}\right)\right)}{1-\tanh ^{2}\left(X_{t}\right)} d t+\frac{\sigma}{\sqrt{1-\tanh ^{2}\left(X_{t}\right)}} d W_{t}, \quad t \geq 0, X_{0}=x_{0}
$$

where $\kappa$ and $\sigma$ are positive, $\mu \in(-1,1)$. Next, we transform (12) with $\rho_{t}=\tanh \left(X_{t}\right)$. Again, applying Itô's Lemma we obtain after a tedious calculation

$$
d \rho_{t}=\left[\left(\kappa\left(\mu-\rho_{t}\right)\right)-\sigma^{2} \rho_{t}\right] d t+\sigma \sqrt{1-\rho_{t}^{2}} d W_{t} .
$$

Next, if we define

$$
\kappa^{*}=\kappa+\sigma^{2}, \quad \mu^{*}=\frac{\kappa \mu}{\kappa+\sigma^{2}}, \quad \sigma^{*}=\sigma
$$

the correlation process (13) can be rewritten as

$$
d \rho_{t}=\kappa^{*}\left(\mu^{*}-\rho_{t}\right) d t+\sigma^{*} \sqrt{1-\rho_{t}^{2}} d W_{t}
$$

which is exactly the van Emmerich's correlation process in [10]. Due to the transformation with the function tanh, the correlations provided by (15), with coefficients (14), are obviously located in the interval $(-1,1)$. We can check this important property in another way: We recall that van Emmerich [10] derived the analytic condition

$$
\kappa^{*} \geq \frac{\sigma^{*}}{1 \pm \mu^{*}}
$$

to ensure that the boundaries -1 and 1 are unattainable. We see that the correlation process (15) must have already satisfied the condition (16): Substituting (14) in (16) we obtain

$$
\frac{\sigma^{2}}{\kappa(1 \pm \mu)+\sigma^{2}} \leq 1
$$

which always holds whilst $\kappa$ is positive and $\mu \in(-1,1)$.

\section{Stochastic correlation with a modified Ornstein-Uhlenbeck process}

In this section, we specify a SCP by a hyperbolic transformation of the modified OrnsteinUhlenbeck process. The derivation of the transition density function of this SCP is provided in a closed form. Then, we analyse this density function and show how to fit the correlation process to the historical market data.

\subsection{The transformed modified Ornstein-Uhlenbeck process}

The Ornstein-Uhlenbeck process is defined by the SDE

$$
d X_{t}=\kappa\left(\mu-X_{t}\right) d t+\sigma d W_{t}
$$

where $\kappa, \sigma>0$ and $X_{0}, \mu \in \mathbb{R}$. If we want to restrict the mean reversion value $\mu$ to be only in $(-1,1)$, it is reasonable to modify the Ornstein-Uhlenbeck process $(18)$ as

$$
d X_{t}=\kappa\left(\mu-\tanh \left(X_{t}\right)\right) d t+\sigma d W_{t},
$$


where $\kappa, \sigma>0$ and $X_{0}, \mu \in(-1,1)$.

Lemma 1 The SCP $\rho_{t}=\tanh \left(X_{t}\right)$ satisfies the $S D E$

$$
d \rho_{t}=\left(1-\rho_{t}^{2}\right)\left(\kappa\left(\mu-\rho_{t}\right)-\sigma^{2} \rho_{t}\right) d t+\left(1-\rho_{t}^{2}\right) \sigma d W_{t},
$$

where $t \geq 0, \rho_{0} \in(-1,1), \kappa, \sigma>0$ and $\mu \in(-1,1)$.

Proof Applying Itô's Lemma we obtain

$$
\begin{aligned}
d \rho_{t} & =\frac{\partial \tanh \left(X_{t}\right)}{\partial x} d X_{t}+\frac{1}{2} \frac{\partial^{2} \tanh \left(X_{t}\right)}{\partial x^{2}} \sigma^{2} d t \\
& =\operatorname{sech}^{2}\left(X_{t}\right) \kappa\left(\mu-\tanh \left(X_{t}\right)\right) d t-\operatorname{sech}^{2}\left(X_{t}\right) \frac{\sinh \left(X_{t}\right)}{\cosh \left(X_{t}\right)} \sigma^{2} d t+\operatorname{sech}^{2}\left(X_{t}\right) \sigma^{2} d W_{t} \\
& =\left(1-\rho_{t}^{2}\right) \kappa\left(\mu-\rho_{t}\right) d t-\left(1-\rho_{t}^{2}\right) \rho_{t} \sigma^{2} d t+\left(1-\rho_{t}^{2}\right) \sigma^{2} d W_{t}=(20) .
\end{aligned}
$$

Let us introduce the notation

$$
\kappa^{*}=\kappa+\sigma^{2}, \quad \mu^{*}=\frac{\kappa \mu}{\kappa+\sigma^{2}}, \quad \sigma^{*}=\sigma .
$$

and rewrite (20) as

$$
\frac{d \rho_{t}}{1-\rho_{t}^{2}}=\kappa^{*}\left(\mu^{*}-\rho_{t}\right) d t+\sigma^{*} d W_{t}
$$

where $t \geq 0, \rho_{0} \in(-1,1), \kappa^{*}, \sigma^{*}>0$ and $\mu^{*} \in(-1,1)$.

\subsection{Transition density function}

For calibration purposes, we first determine the transition density function of (22) with the aid of the Fokker-Planck equation [15]. Then, we obtain the parameters of the correlation process (22) by fitting the density function to the market data.

Let us assume that the $\operatorname{SCP}(22)$ possesses a transition density $f\left(t, \tilde{\rho} \mid \rho_{0}\right)$ which satisfies the following Fokker-Planck equation

$$
\frac{\partial}{\partial t} f(t, \tilde{\rho})+\frac{\partial}{\partial \tilde{\rho}}(\hat{a}(t, \tilde{\rho}) f(t, \tilde{\rho}))-\frac{1}{2} \frac{\partial^{2}}{\partial \tilde{\rho}^{2}}\left(\hat{b}(t, \tilde{\rho})^{2} f(t, \tilde{\rho})\right)=0,
$$

with

$$
\hat{a}(t, \tilde{\rho})=\kappa^{*}\left(\mu^{*}-\tilde{\rho}\right)\left(1-\tilde{\rho}^{2}\right), \quad \hat{b}(t, \tilde{\rho})=\left(1-\tilde{\rho}^{2}\right) \sigma^{*} .
$$

For the calibration purpose we consider the stationary density (for $t \rightarrow \infty$ )

$$
f(\tilde{\rho}):=\lim _{t \rightarrow \infty} f\left(t, \tilde{\rho} \mid \rho_{0}\right) .
$$

With the above construction the SCP (22) is also a mean-reverting process. Thus one can show that every two solutions of (23) are the same for $t \rightarrow \infty$, i.e. a unique stationary solution $f(\tilde{\rho})$ exists, cf. [15]. 
In the sequel, we show how to determine the analytical stationary density function $f(\tilde{\rho})$ of the SCP (22). First, the stationary density function $f(\tilde{\rho})$ obviously satisfies

$$
\frac{\partial}{\partial \tilde{\rho}}\left(\left(1-\tilde{\rho}^{2}\right)\left(\kappa^{*}\left(\mu^{*}-\tilde{\rho}\right)\right) f(\tilde{\rho})\right)=\frac{1}{2} \frac{\partial^{2}}{\partial \tilde{\rho}^{2}}\left(\left(1-\tilde{\rho}^{2}\right) \sigma^{*}\right)^{2} f(\tilde{\rho}) .
$$

By solving the elliptic equation (26) with the aid of using Maple we obtain the stationary $\operatorname{density} f(\tilde{\rho})$ as

$$
\begin{aligned}
f(\tilde{\rho})= & \frac{m}{2^{\frac{\kappa^{*}}{\sigma^{*}}}}(1+\tilde{\rho})^{\frac{\kappa^{*}-2 \sigma^{* 2}}{\sigma^{* 2}}+\frac{\kappa^{*} \mu^{*}}{\sigma^{* 2}}}(1-\tilde{\rho})^{\frac{\kappa^{*}-2 \sigma^{* 2}}{\sigma^{* 2}}-\frac{\kappa^{*} \mu^{*}}{\sigma^{* 2}}} \\
& +\frac{n}{\tilde{\rho}^{2}-1}\left(\frac{1}{2}\right)^{\frac{2 \sigma^{* 2}-\kappa^{*}}{\sigma^{* 2}}} F\left(1, \frac{2 \sigma^{* 2}-2 \kappa^{*}}{\sigma^{* 2}}, \frac{\left(-\mu^{*}-1\right) \kappa^{*}+2 \sigma^{* 2}}{\sigma^{* 2}}, \frac{\tilde{\rho}}{2}+\frac{1}{2}\right)
\end{aligned}
$$

with the constants $m, n \in \mathbb{R}$ and the hypergeometric function $F$ which is defined as

$$
F(a, b, c, x)=\sum_{k=0}^{\infty} \frac{x^{k}}{k !} \frac{(a)_{k}(b)_{k}}{(c)_{k}}, \quad|x|<1,
$$

where $(\cdot)_{k}$ denotes the Pochhammer symbol,

$$
(a)_{k}=a(a+1)(a+2) \cdots(a+k-1), \quad(a)_{0}=1 .
$$

Next we need to fix the constants $m$ and $n$ in (27) to obtain the stationary density. Due to the mean reversion the stationary $\operatorname{density} f(\tilde{\rho})$ must satisfy

$$
\int_{-1}^{1} \tilde{\rho} f(\tilde{\rho}) d \tilde{\rho}=\mu^{*}
$$

If we choose $\mu^{*}=0$, we observe that the first term in (27) becomes

$$
\frac{m}{2^{\frac{\kappa^{*}}{\sigma^{* 2}}}}(1+\tilde{\rho})^{\frac{\kappa^{*}-2 \sigma^{* 2}}{\sigma^{* 2}}}(1-\tilde{\rho})^{\frac{\kappa^{*}-2 \sigma^{* 2}}{\sigma^{* 2}}},
$$

which is obviously symmetric around $\tilde{\rho}=0$, i.e. the condition (30) will be fulfilled for $n=0$. In the sequel we assume that $n \equiv 0$ for all general $\mu^{*} \in(-1,1)$ such that the transition density function (27) can be rewritten as

$$
f(\tilde{\rho})=\frac{m}{2^{\frac{\kappa^{*}}{\sigma^{* 2}}}}(1+\tilde{\rho})^{\frac{\kappa^{*}-2 \sigma^{* 2}}{\sigma^{* 2}}+\frac{\kappa^{*} \mu^{*}}{\sigma^{* 2}}}(1-\tilde{\rho})^{\frac{\kappa^{*}-2 \sigma^{* 2}}{\sigma^{* 2}}-\frac{\kappa^{*} \mu^{*}}{\sigma^{* 2}}} .
$$

To determine the value of $m$ we can employ the basic property of a density function

$$
\int_{-1}^{1} f(\tilde{\rho}) d \tilde{\rho}=1
$$

The constant $m$ in (31) must be chosen such that the normalization condition (32) is always fulfilled. We set

$$
a=\frac{\kappa^{*}-2 \sigma^{* 2}}{\sigma^{* 2}}, \quad b=\frac{\kappa^{*} \mu^{*}}{\sigma^{* 2}}
$$


and substitute it into (31) to obtain

$$
f(\tilde{\rho})=\frac{m}{2^{\kappa^{*}}}(1+\tilde{\rho})^{a+b}(1-\tilde{\rho})^{a-b}
$$

As long as

$$
a \pm b>-1
$$

the integral

$$
\int_{-1}^{1}(1+\tilde{\rho})^{a+b}(1-\tilde{\rho})^{a-b} d \tilde{\rho}
$$

has the solution

$$
\begin{aligned}
M:= & \frac{\Gamma(1+a-b) F(1,-a-b, 2+a-b,-1)}{\Gamma(2+a-b)} \\
& +\frac{\Gamma(1+a+b) F(1,-a+b, 2+a+b,-1)}{\Gamma(2+a+b)},
\end{aligned}
$$

with the hypergeometric function $F$ defined in (28) and the gamma function $\Gamma$.

Next we show that if $\mu \in(-1,1)$ then (35) holds. Using the definitions of $\kappa^{*}, \mu^{*}$ and $\sigma^{*}$ in (21) and together with (33) we obtain

$$
a=\frac{\kappa^{*}-2 \sigma^{* 2}}{\sigma^{* 2}}=\frac{\kappa-\sigma^{2}}{\sigma^{2}}, \quad b=\frac{\kappa^{*} \mu^{*}}{\sigma^{* 2}}=\frac{\kappa \mu}{\sigma^{2}} .
$$

We consider the following simple calculations

$$
\begin{aligned}
& \mu>-1 \Rightarrow \kappa(1+\mu)>0 \Rightarrow \frac{\kappa-\sigma^{2}}{\sigma^{2}}+\frac{\kappa \mu}{\sigma^{2}}>-1 \Rightarrow a+b>-1, \\
& \mu<1 \Rightarrow \kappa(1-\mu)>0 \Rightarrow \frac{\kappa-\sigma^{2}}{\sigma^{2}}-\frac{\kappa \mu}{\sigma^{2}}>-1 \Rightarrow a-b>-1
\end{aligned}
$$

and realize that the condition (35) will always hold due to $\mu \in(-1,1)$. Thus, the constant $m$ can be determined as

$$
m=\frac{2^{\frac{\kappa^{*}}{\sigma^{* 2}}}}{M} .
$$

Finally, we obtain the transition density function in a closed form as

$$
f(\tilde{\rho})=\frac{(1+\tilde{\rho})^{a+b}(1-\tilde{\rho})^{a-b}}{M}
$$

with $a, b$ defined in (33) and $M$ in (36). The parameters $\kappa^{*}, \mu^{*}$ and $\sigma^{*}$, or rather, $\kappa, \mu$ and $\sigma$ can be obtained by fitting the expression (39) to the historical correlation from market data, see Section 3.3. 
We could generalize the correlation process (22) with the same definition but directly with the arbitrary parameter coefficients $\kappa>0, \mu \in(-1,1)$ and $\sigma>0$, like

$$
\frac{d \rho_{t}}{1-\rho_{t}^{2}}=\kappa\left(\mu-\rho_{t}\right) d t+\sigma d W_{t}
$$

For this case, we have for $a$ and $b$, as defined in (33), as

$$
a=\frac{\kappa-2 \sigma^{2}}{\sigma^{2}}, \quad b=\frac{\kappa \mu}{\sigma^{2}} .
$$

We perform a similar calculation for checking the condition (35) as above:

$$
\begin{aligned}
& a+b>-1 \Leftarrow \frac{\kappa-2 \sigma^{2}}{\sigma^{2}}+\frac{\kappa \mu}{\sigma^{2}}>-1 \Leftarrow \kappa(1+\mu)>\sigma^{2} \Leftarrow \kappa>\frac{\sigma^{2}}{1+\mu}, \\
& a-b>-1 \Leftarrow \frac{\kappa-2 \sigma^{2}}{\sigma^{2}}-\frac{\kappa \mu}{\sigma^{2}}>-1 \Leftarrow \kappa(1-\mu)>\sigma^{2} \Leftarrow \kappa>\frac{\sigma^{2}}{1-\mu} .
\end{aligned}
$$

Thus, the process (40) could be employed for the stochastic correlation if the condition

$$
\kappa>\frac{\sigma^{2}}{1 \pm \mu}
$$

is fulfilled. We find that this condition dovetails nicely with that condition in [10], which ensures that the boundaries -1 and 1 are unattainable.

To further illustrate the transition density function $f(\tilde{\rho})$, we display in Figures 4,5 and 6 the behaviour of $f(\tilde{\rho})$ for different values of each parameter. In Figure 4 , we let $\kappa=2$ and $\mu=0$ and display $f(\tilde{\rho}) i$ th different values of $\sigma$, which is equal to $0.3,0.4$ and 0.5 , respectively. Obviously, $\sigma$ shows the magnitude of variation from the mean value $\mu=0$. Next, we fix $\kappa=2$ and $\sigma=0.3$, the behaviour of $f(\tilde{\rho})$ only with varying mean value $\mu=-0.5, \mu=0$ and $\mu=0.5$ can be found in Figure 5 . However, whilst $\mu=-0.5$ and $\mu=0.5$ we can observe that the peak of the corresponding $f(\tilde{\rho})$ does not locate exactly at the points $\tilde{\rho}=-0.5$ and $\tilde{\rho}=0.5$, respectively. The reason is that, the value of $\kappa$, which is mean reversion rate, is not large enough. In order to illustrate the role of $\kappa$, we set $\mu=-0.5, \sigma=0.5$ and vary the value

Figure 4 Comparison of $f(\tilde{\rho})$ for different values of $\sigma(\kappa=2$ and $\mu=0)$.

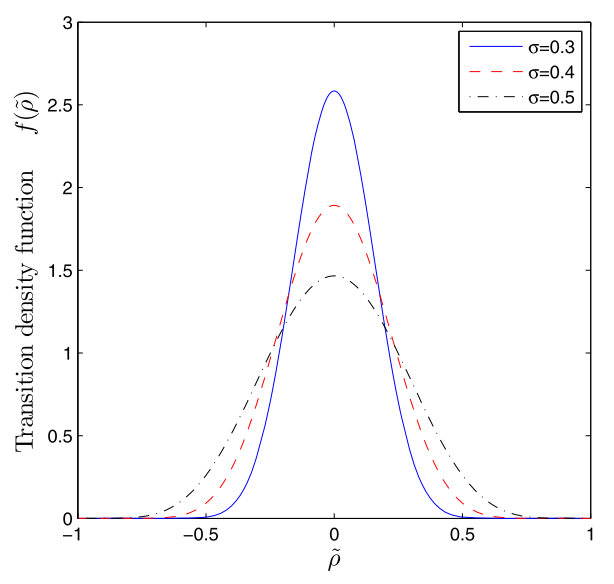


Figure 5 Comparison of $f(\tilde{\rho})$ for different values of $\mu$ ( $\kappa=2$ and $\sigma=0.3$ ).

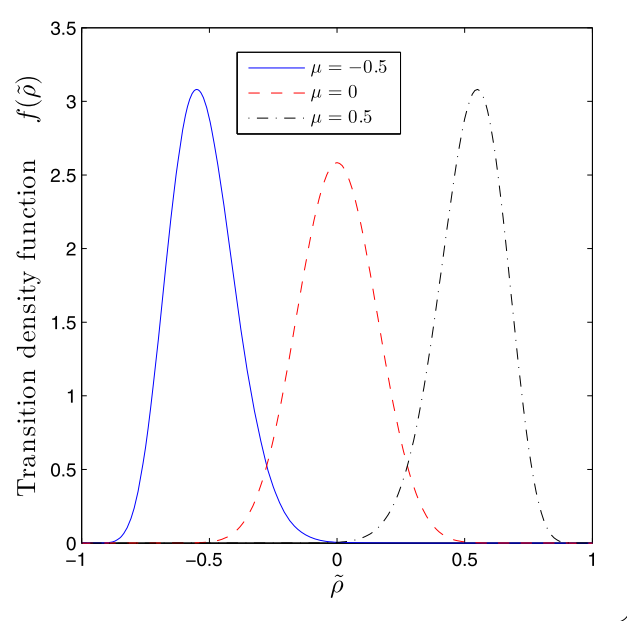

Figure 6 Comparison of $f(\tilde{\rho})$ for different values of $\kappa(\mu=-0.5$ and $\sigma=0.5)$.

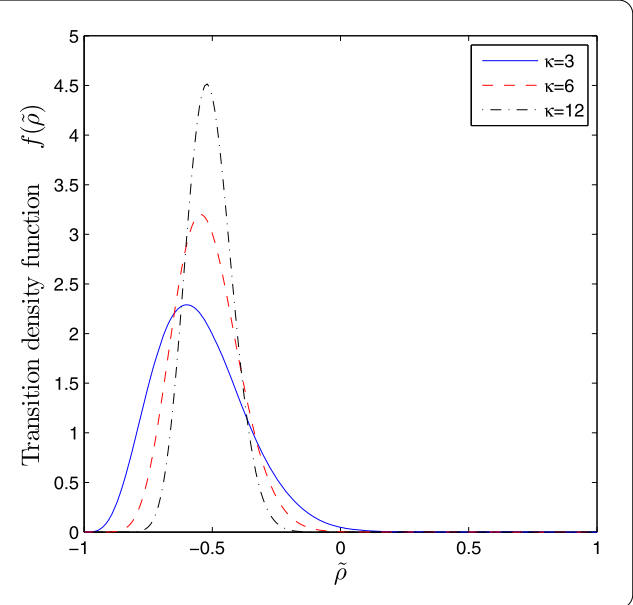

of $\kappa$, see Figure 6 . For $\kappa=3$, the peak of the transition density function is far away from the mean value -0.5 . However, in contrast the peak reaches already the point $\tilde{\rho}=-0.5$ when $\kappa=12$.

\subsection{Calibration}

We assume that the correlation is itself observable. Under this assumption the transition density can be used for calibration purposes. One uses usually maximum-likelihood estimation (MLE) when the density function is known. Considering the density function (39), it will be tedious to determine its likelihood-function.

Another approach to estimate the parameters is to fit the empirically observed density to the stationary density (39). As an example we fit the historical data from Figures 2 a to (39). The fitting by nonlinear least-squares works well, see Figure 7.

\section{Stochastically correlated Brownian motions}

The remaining problem is how to incorporate the stochastic correlation process in the financial model, e.g. how to use the stochastic correlation in the option pricing model. In Section 1, we mentioned that a widely used approach for dependence is to consider the (constant) correlated Brownian motions. In order to consider a stochastic correlation, we 


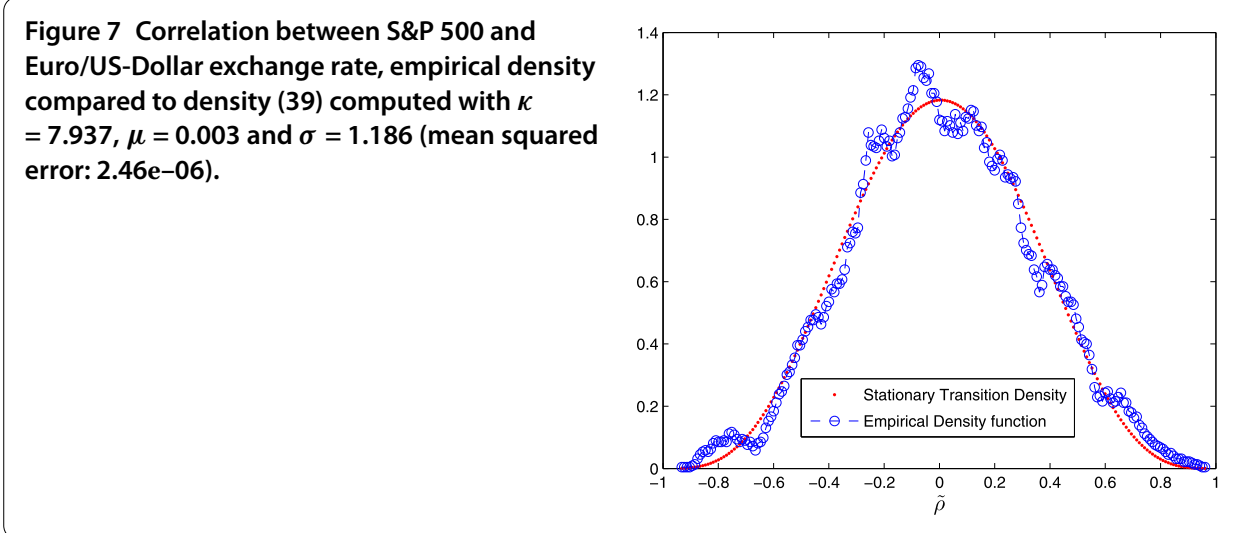

need the concept of stochastically correlated Brownian motions. In the following, we study the stochastically correlated Brownian motions following the work of van Emmerich [10].

Based on two independent Brownian motions $W_{2, t}$ and $W_{3, t}$ we define

$$
W_{1, t}=\int_{0}^{t} \rho_{s} d W_{2, s}+\int_{0}^{t} \sqrt{1-\rho_{s}^{2}} d W_{3, s}
$$

where $\rho_{t}$ is one SCP of type (10), and we assume that $W_{t}$ in (10) is independent of each $W_{i, t}$, for $i=2,3$.

\section{Lemma $2 W_{1, t}$ satisfies}

(1) $W_{1,0}=0$,

(2) $\mathbb{E}\left[\left(W_{1, t}\right)^{2}\right]=t$,

(3) $\mathbb{E}\left[W_{1, t} \mid \mathcal{F}_{s}\right]=W_{1, s}$, for $s \leq t$.

Proof (1) is obvious. We calculate the two expected values as follows:

$$
\begin{aligned}
\mathbb{E}\left[\left(W_{1, t}\right)^{2}\right]= & \mathbb{E}\left[\left(\int_{0}^{t} \rho_{s} d W_{2, s}\right)^{2}+\left(\int_{0}^{t} \sqrt{1-\rho_{s}^{2}} d W_{3, s}\right)^{2}\right. \\
& \left.+2 \int_{0}^{t} \rho_{s} d W_{2, s} \int_{0}^{t} \sqrt{1-\rho_{s}^{2}} d W_{3, s}\right] \\
= & \mathbb{E}\left[\int_{0}^{t} \rho_{s}^{2} d s+\int_{0}^{t}\left(1-\rho_{s}^{2}\right) d s\right]+\underbrace{\mathbb{E}\left[2 \int_{0}^{t} \rho_{s} d W_{2, s} \int_{0}^{t} \sqrt{1-\rho_{s}^{2}} d W_{3, s}\right]}_{=0, \text { since } W_{2} \perp W_{3}} \\
= & \int_{0}^{t} 1 d s=t, \\
\mathbb{E}\left[W_{1, t} \mid \mathcal{F}_{s}\right]= & W_{1, s}+\underbrace{\mathbb{E}\left[\int_{s}^{t} \rho_{s_{1}} d W_{2, s_{1}}+\int_{s}^{t} \sqrt{1-\rho_{s_{1}}^{2}} d W_{3, s_{1}} \mid \mathcal{F}_{s}\right]}_{:=0} .
\end{aligned}
$$

This means that we have defined one new Brownian motion $W_{1, t}$ regarding the two independent Brownian motion $W_{2, t}$ and $W_{3, t}$. Besides, we can check that

$$
\mathbb{E}\left[W_{1, t} \cdot W_{2, t}\right]=\mathbb{E}\left[\int_{0}^{t} \rho_{s} d s\right]
$$


which is the definition for the case that the Brownian motions $W_{1, t}$ and $W_{2, t}$ are correlated by the SCP $\rho_{t}$. One can immediately see that (44) agrees for

$$
\mathbb{E}\left[W_{1, t} \cdot W_{2, t}\right]=\rho t,
$$

where $W_{1, t}$ and $W_{2, t}$ are correlated by the constant $\rho$. Indeed, (44) can be also seen as that $W_{1, t}$ and $W_{2, t}$ are correlated by the average correlation

$$
\frac{1}{t} \int_{0}^{t} \mathbb{E}\left[\rho_{s}\right] d s
$$

which is a constant.

\section{Pricing quantos with stochastic correlation}

To illustrate the impact of using stochastic correlation on option pricing, we use quanto options as an example. These options hedge the exchange rate risk when investing in financial products not valued in the domestic currency. To price these options, one has to consider the correlation between the currency exchange rate $R_{t}$ between domestic and foreign currencies, and the price $S_{t}$ of the underlying. We assume that $S_{t}$ and $R_{t}$ satisfy the SDEs

$$
\left\{\begin{array}{l}
d S_{t}=\mu_{S} S_{t} d t+\sigma_{S} S_{t} d W_{t}^{S}, \\
d R_{t}=\mu_{R} R_{t} d t+\sigma_{R} R_{t} d W_{t}^{R},
\end{array}\right.
$$

where $W_{t}^{S}$ and $W_{t}^{R}$ are correlated using the SCP (40) as:

$$
\frac{d \rho_{t}}{1-\rho_{t}^{2}}=\kappa\left(\mu-\rho_{t}\right) d t+\sigma d W_{t}
$$

$W_{t}$ is assumed to be independent of $W_{t}^{S}$ and $W_{t}^{R}$.

We consider as an example a Put-Option on the S\&P 500 with payoff in Euro [12]

$$
(\underbrace{\text { Strike }}_{:=K}-\underbrace{\mathrm{S} \& \mathrm{P} 500_{T}}_{:=S_{T}})^{+}
$$

where $(\cdot)^{+}=\max (0, \cdot)$. Then the payoff in US-Dollar can be written with the Euro/USDollar exchange rate as

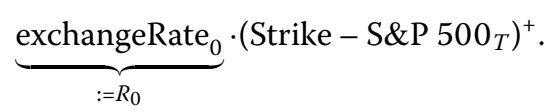

We denote the risk-free interest rate of Euro and US-Dollar respectively by $r_{e}$ and $r_{d}$. If $W_{t}^{S}$ and $W_{t}^{R}$ are correlated with a constant correlation, the price of a Quanto Put-Option in the Black-Scholes (BS) model with continuous dividend yield is [16]:

$$
P_{\text {Quanto }}\left(S_{0}, K, r_{e}, r_{d}, D, \sigma_{S}, \sigma_{R}, T\right)=R_{0}\left(K \exp ^{-r_{d} T} \mathcal{N}\left(-d_{2}\right)-S_{0} \exp ^{-D T} \mathcal{N}\left(-d_{1}\right)\right)
$$


with

$$
d_{1}=\frac{\log \left(\frac{S_{0}}{K}\right)+\left(\left(r_{d}-D\right)+\frac{\sigma_{S}^{2}}{2}\right) / T}{\sigma_{S} \sqrt{T}}, \quad d_{2}=d_{1}-\sigma_{S} \sqrt{T}, \quad D=r_{d}-r_{e}+\rho \sigma_{S} \sigma_{R}
$$

We follow the train of thoughts in van Emmerich [10] to incorporate the stochasticity of the correlation in the BS price. The no-arbitrage principle requires

$$
\frac{1}{R_{0}} \exp \left(r_{e} T\right) \mathbb{E}\left[R_{T}\right]=\exp \left(r_{d} T\right)
$$

and

$$
\frac{1}{R_{0}} \frac{1}{S_{0}} \mathbb{E}\left[S_{T} R_{T}\right]=\exp \left(r_{d} T\right)
$$

(49) can be interpreted as: The expected return of one unit of US-Dollar, exchanged to Euro, risk-free invested in the Euro countries and re-exchanged to US-Dollar must equal the risk-free return on one unit of US-Dollar in US-Dollar countries. The interpretation of (50) is analogous, the left side of (50) describes the re-exchanged expected value of an investment of one US-Dollar into the underlying with price $S$. Further computing (49) and (50) by aid of Itô’s lemma we obtain

$$
\mu_{R}=r_{d}-r_{e}
$$

and

$$
\mu_{S}=r_{d}-\mu_{R}-\sigma_{S} \sigma_{R} \frac{1}{T} \int_{0}^{T} \rho_{t} d t
$$

In the BS model, we interpret (52) as a return minus the continuous dividend

$$
D\left(\rho_{t}\right):=\mu_{R}+\sigma_{S} \sigma_{R} \frac{1}{T} \int_{0}^{T} \rho_{t} d t=r_{d}-r_{e}+\sigma_{S} \sigma_{R} \frac{1}{T} \int_{0}^{T} \rho_{t} d t
$$

The integral of the stochastic correlation $\rho_{t}$ can be computed numerically using e.g. the Milstein scheme [17]. Finally, the price of a Quanto Put-Option in the extended BS model incorporating the SCP reads

$$
\begin{aligned}
P_{\text {Quanto }} & =P_{\text {Quanto }}\left(S_{0}, K, r_{e}, r_{d}, D\left(\rho_{t}\right), \sigma_{S}, \sigma_{R}, T\right) \\
& =R_{0}\left(K \exp ^{-r_{d} T} \mathcal{N}\left(-d_{2}\right)-S_{0} \exp ^{-D\left(\rho_{t}\right) T} \mathcal{N}\left(-d_{1}\right)\right)
\end{aligned}
$$

with

$$
d_{1}=\frac{\log \left(\frac{S_{0}}{K}\right)+\left(\left(r_{d}-D\left(\rho_{t}\right)\right)+\frac{\sigma_{S}^{2}}{2}\right) / T}{\sigma_{S} \sqrt{T}}, \quad d_{2}=d_{1}-\sigma_{S} \sqrt{T} .
$$

The price of a Quanto Call-Option is derived easily from the put-call parity [16]. 


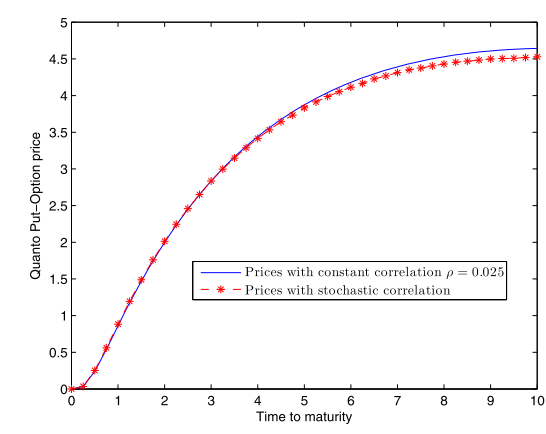

(a) Comparison of prices between using stochastic and constant correlation

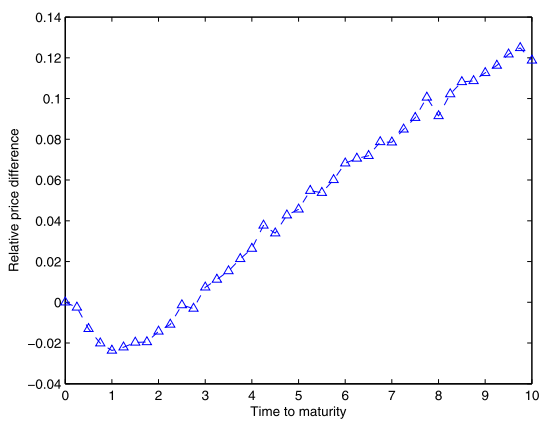

(b) Relative price difference

Figure 8 BS parameters: $K=80, S_{0}=100, R_{0}=1, r_{d}=0.05, r_{e}=0.03, \sigma_{S}=0.2, \sigma_{R}=0.4, \mathrm{SCP}$ parameters: $\kappa=7.937, \mu=0.003, \sigma=1.186$ and $\rho_{0}=0.3$.

We use a conditional Monte-Carlo approach and first simulate all the paths of $\rho_{t}^{i}$, for $i \in\{1,2, \ldots, M\}$ and for each path we can compute a price $P_{\text {Quanto }}^{i}$ by the pricing formula (53). Then the fair price $\mathbb{P}_{0}$ is the mean value over all prices

$$
\mathbb{P}_{0}=\mathbb{E}\left[\mathbb{E}\left[P_{\text {Quanto }} \mid \mathcal{F}_{t}\right]\right] \approx \frac{\sum_{i=1}^{M} P_{\text {Quanto }}^{i}}{M} .
$$

In Figure 8, we assume the parameter for the Black-Scholes model and use the estimated parameter for the SCP model (see Figure 7). Besides, we apply the sample coefficient correlation (3) to estimate a constant correlation using the whole historical data (Jan 2003-Mar 2013) of S\&P 500 and Euro/US-Dollar exchange rate, which is 0.025. At the same time, we can let the SCP starting from the first correlation in the historical correlations. In Figure $8 \mathrm{~b}$ we present the relative difference between the price with constant correlation and stochastic correlation. We can observe, whilst the maturity $T$ is shorter than three years, the prices with constant correlation are lower than the prices with stochastic correlation. However, for the contracts with maturities which are longer than three years, the prices using constant correlation are higher than the prices using stochastic correlation. The reason for this, for a short maturity $(T<3)$, the SCP provides the correlations which are still closed to the initial correlation $\rho_{0}=0.3$, which is larger than the constant correlation $\rho=0.025$. Since the price of quanto increases direct proportional with the correlation, therefore, the prices using stochastic correlation are higher than the prices using constant correlation for these short maturities. However, for the long maturities $(T>3)$, the generated correlations will tend to the mean reversion value $\mu=0.003$ which are less than the value of the constant correlation, the prices with the constant correlation are thus higher.

If we let $\rho_{0}$ to be a value which is lower than the constant correlation $\rho=0.025$, e.g. $\rho_{0}=-0.6$. And we choose a larger value for $\mu$, say 0.1 . We can expect that the prices with constant correlation will be higher than the prices with stochastic correlation only for the short maturities. For the longer maturities, the prices using stochastic correlations are higher, see Figures 9.

Furthermore, to illustrate the role of the parameters $\kappa$ and $\sigma$ in pricing a Quanto option, we display the prices by varying the values of $\kappa$ and $\sigma$ in Figure 10a and 10b, respectively. In Figure 10a we see that the both prices using constant and stochastic correlation are quite 


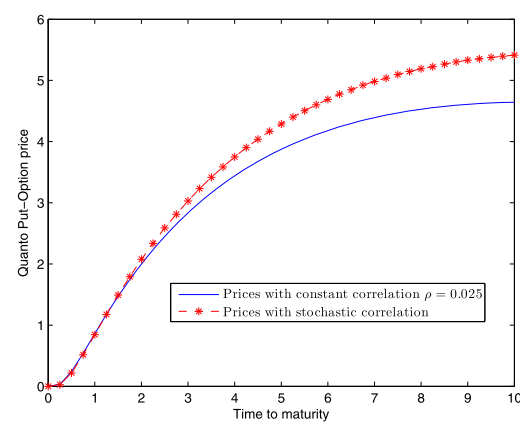

(a) Comparison of prices between using stochastic and constant correlation

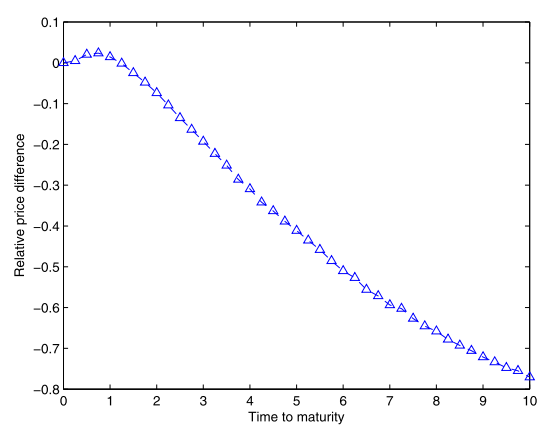

(b) Relative price difference

Figure 9 BS parameters: $K=80, S_{0}=100, R_{0}=1, r_{d}=0.05, r_{e}=0.03, \sigma_{S}=0.2, \sigma_{R}=0.4, \mathrm{SCP}$ parameters: $\kappa=7.937, \mu=0.1, \sigma=1.186$ and $\rho_{0}=-0.6$.
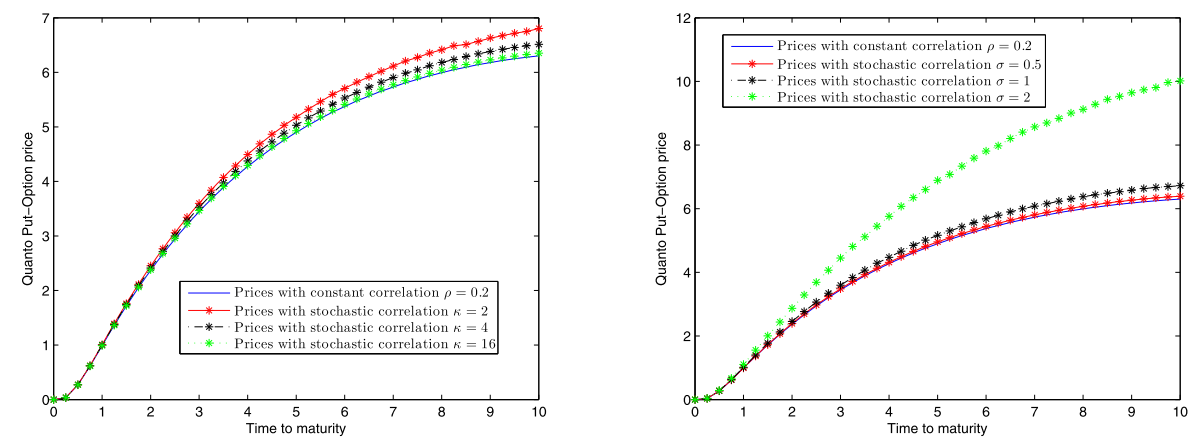

Figure $10 \mathrm{BS}$ parameters: $K=80, S_{0}=100, R_{0}=1, r_{d}=0.05, r_{e}=0.03, \sigma_{S}=0.2, \sigma_{R}=0.4$.

closed to each other for $\kappa=16$. This is because that, for a large value of $\kappa$, the correlation tends rapidly to the mean reversion value $\mu$ of the SCP process. And the value of $\mu$ has been set to be equal to the value of constant correlation, the price differences are thus quite small.

In contrast, fixing a value for $\kappa$, the price differences between using constant and stochastic correlation become bigger by increasing the value of the diffusion $\sigma$ (and thus randomness in the SCP process), as shown in Figure 10b.

\section{Conclusion}

In this work we have revised concisely some stochastic correlation models. Market observations give strong evidence that financial quantities are correlated in a strongly nonlinear, non-deterministic way. Instead of assuming a constant correlation, correlation has to be modelled as a stochastic process. We discussed first the general stochastic correlation model proposed in [12] and proved that the stochastic correlation process in [10] can be obtained by applying this general approach.

We generalized our approach [12] to derive a stochastic correlation model from a hyperbolic transformation of the modified Ornstein-Uhlenbeck process allowing for a transition density function in a closed form and an easy-to-handle calibration to historical data. As an example, we computed the fair price of a Quanto Put-option with stochastic cor- 
relation. The numerical results showed that the correlation risk caused by using a wrong (constant) correlation model cannot be neglected.

\section{Competing interests}

The authors declare that they have no competing interests

\section{Authors' contributions}

All authors contributed to this paper as a whole. However, special merits go to LT for the idea and careful analysis; to ME for the introduction and MG for the application example. All authors read and approved the final manuscript.

\section{Acknowledgements}

The work of the authors was partially supported by the European Union in the FP7-PEOPLE-2012-ITN Programme under Grant Agreement Number 304617 (FP7 Marie Curie Action, Project Multi-ITN STRIKE - Novel Methods in Computational Finance). Further the authors acknowledge partial support from the bilateral German-Spanish Project HiPeCa-High Performance Calibration and Computation in Finance, financed by DAAD.

Received: 16 November 2015 Accepted: 2 March 2016 Published online: 15 March 2016

\section{References}

1. Campbell JW, Lo AW, MacKinlay AC. The econometrics of financial markets. Princeton: Princeton University Press: 1997.

2. Brigo D, Chourdakis K. Counterparty risk for credit default swaps: impact of spread volatility and default correlation. Int J Theor Appl Finance. 2009;12:1007-26.

3. Teng L, Ehrhardt M, Günther M. Bilateral counterparty risk valuation of CDS contracts with simultaneous defaults. Int J Theor Appl Finance. 2013;16(7):1350040.

4. McNeil AJ, Frey R, Embrechts P. Quantitative risk management: concepts, techniques, and tools. Princeton: Princeton University Press; 2005.

5. Schöbel R, Zhu J. Stochastic volatility with an Ornstein-Uhlenbeck process: an extension. Eur Finance Rev. 1999:3:23-46.

6. Engle RF. Dynamic conditional correlation: a simple class of multivariate GARCH. J Bus Econ Stat. 2002;20(3):339-50.

7. Langnau A. Introduction into "local correlation" modelling. arXiv:0909.3441 (2009)

8. Gourieroux C, Jasiak J, Sufana R. The Wishart autoregressive process of multivariate stochastic volatility. J Econom. 2009;150:167-81.

9. Bowman AW, Azzalini A. Applied smoothing techniques for data analysis. New York: Oxford University Press; 1997.

10. van Emmerich C. Modelling correlation as a stochastic process. Preprint 06/03, University of Wuppertal (June 2006)

11. Ma J. Pricing foreign equity options with stochastic correlation and volatility. Ann Econ Financ. 2009;10(2):303-27.

12. Teng L, van Emmerich C, Ehrhardt M, Günther M. A versatile approach for stochastic correlation using hyperbolic functions. Int J Comput Math. 2016;93(3):524-39.

13. Oksendal B. Stochastic differential equations. Berlin: Springer; 2000

14. Uhlenbeck GE, Ornstein LS. On the theory of Brownian motion. Phys Rev. 1930;36:823-41.

15. Risken H. The Fokker-Planck equation. Berlin: Springer; 1989.

16. Wilmott P. Paul Wilmott on quantitative finance. 2nd ed. West Sussex: Wiley; 2006.

17. Kloeden PE, Platen E. Numerical solution of stochastic differential equations. Berlin: Springer; 1992.

\section{Submit your manuscript to a SpringerOpen ${ }^{\circ}$ journal and benefit from:}

- Convenient online submission

Rigorous peer review

- Immediate publication on acceptance

- Open access: articles freely available online

- High visibility within the field

- Retaining the copyright to your article 